\title{
Determination of Contrast Sensitivity for Mammographic Softcopy Reading
}

\author{
Dörte Apelt, Heinz-Otto Peitgen \\ MeVis Research GmbH, Bremen \\ doerte.apelt@mevis.de
}

\begin{abstract}
Research of visual perception of luminance differences is an important basis for understanding the perception of complex patterns. We introduce a new approach for determining the contrast sensitivity function (CSF) in front of the complex anatomical structures of mammograms. For this purpose a sinusoidal pattern and digits are used as target items. The approximation to contrast thresholds is done by a psychophysical staircase procedure and is performed for a selection of spatial frequencies.
\end{abstract}

\section{Introduction}

The processing of contrast is a fundamental element in the visual pattern recognition. The quality of perceived structural properties of a mammogram depends on the presentation of the images, both from the technical parameters (size, maximum contrast of the monitor, gray value depth etc.) and ambient conditions (e.g. illuminance), and on the visualization method.

In psychological perception research contrast sensitivity is mainly investigated by use of sinusoidal patterns in front of a homogeneous image background [1]. It is not clear how results of those investigations attend to contrast sensitivity in front of a complex background.

In mammography the mapping of luminance differences is studied by test patterns like CDMAM phantoms [2] or by simulated or extracted mammographic lesions [3], and appropriate contrast detail diagrams. The CDMAM phantom permits a standardized assessment of the imaging properties of a system, although it disregards the complex anatomical structure of mammograms. On the other hand the use of lesions in different contrast levels together with a mammogram takes into account anatomical noise, but has the disadvantage that the results of an investigation strongly depend on the radiological expertise of the observers.

In this paper an approach is presented, that combines the advantages of using a standardized test pattern with that of a realistic complex mammographic image background. 


\section{Material and Methods}

\subsection{Target Items: Gabor Patterns and Digits}

For the discrimination task a Gabor pattern is presented in a mammogram on an area of about 2.5 cycles per degree (visual angle) and for a time of $720 \mathrm{~ms}$ (Fig. 1). Contrast, orientation and spatial frequency of the pattern are varied. For the identification task the observer is confronted with digits as target items. The location of the items is marked by a fixation circle. The task of the observer is to focus on the given areal and to pay attention to given properties of the target item. After every presentation of the item the observer has to state the perceived orientation respectively digit. Each measurement starts with a significantly above threshold contrast. Dependent on the observers' answer the contrast is increased or decreased by a defined step width. The observer has to decide between 4 possible orientations respectively 10 possible digits, but furthermore he has the option to answer nothing seen (Unforced Choice Task, UWUD procedure [4]).

During the experiment the contrast is modified by a factor $f>0$, which results in a change of the amplitude. For every pixel the factor marks the difference between the target item and the background.

\subsection{Hypotheses}

Three hypotheses are examined in the experiment:

- A CSF that is determined with a homogeneous background does not allow implications for a CSF with a complex image background, because the complex background involves higher cognitive processing of the visual input (tested for the discrimination task).

- The shape of the CSF is similar for mammograms with different tissue characteristics (tested for the discrimination task).

- The CSF for Gabor patterns (discrimination task) has a higher level than the CSF for digits (identification task).
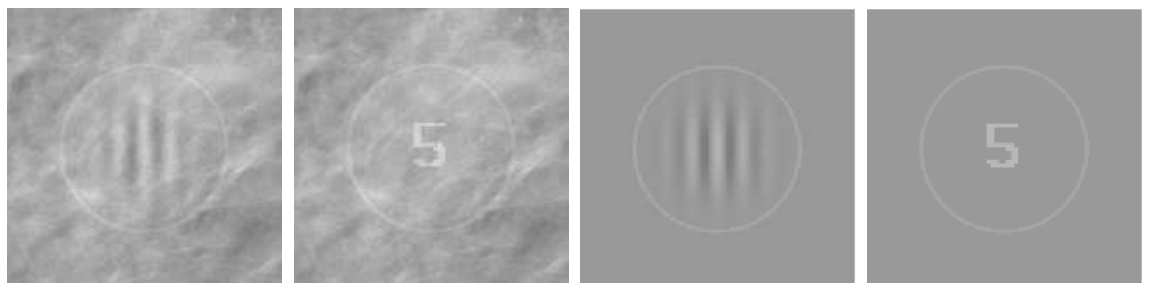

Fig. 1. Parts of the presented images with examples of the used target items (Gabor pattern, digit) with a mammographic respectively homogeneous background 


\subsection{Observers and Experimental Setup}

Eight observers without radiological qualification participated in the experiment. The age range was between 25 and 65 years. The experiment was arranged in a room at an illumination of $10 \mathrm{~lx}$ (measured from viewers position with Gossen MavoLux 5032C). The observer was sitting in circa $57 \mathrm{~cm}$ distance of two $5 \mathrm{~K}$ grayscale displays. On the left screen the target item was presented. The mammogram and the position of the item were not modified during the course of the measurement. Moreover the window level settings were fixed.

In the first 15 minutes measurements of contrast thresholds were conducted ongoing, to follow the adaptation to the luminance. For the last measurement in this period a uniform adaptation level of the observers can be expected (though individual differences in the reached adaptation levels can not be excluded). This measurement was used to check the hypotheses.

The determination of contrast thresholds was done with different images. Fig. 2 shows the corresponding parts of the images, where the items were presented.

\section{$3 \quad$ Results}

The main results of the experiment are shown graphically in Fig. 3, 4 and 5 . Fig. 3 shows the contrast sensitivity functions of two observers P1 and P2 for a homogeneous and a mammographic image background. In both cases the CSF on homogeneous background is higher than on tissue. The contrast sensitivity functions of the same two observers but for different tissues are given in Fig. 4. The difference in contrast sensitivity for Gabor pattern and digits is shown in Fig. 5 .

\section{Discussion}

For all observers the contrast sensitivity is significantly higher on a homogeneous background than on a tissue background with its anatomical noise. An explicit statement regarding the level and the shape of the CSF on tissue can not be derived from the CSF on homogeneous background. On tissue the CSF is less uniform (Fig. 3).
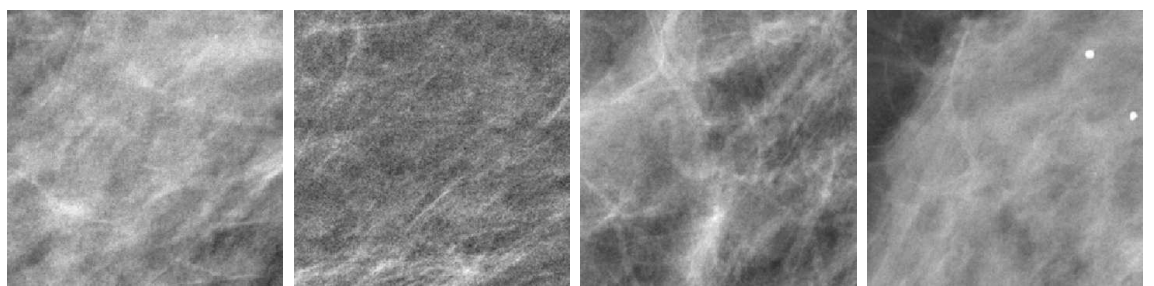

Fig. 2. Parts of the four presented mammograms with different properties in the structure of tissue 

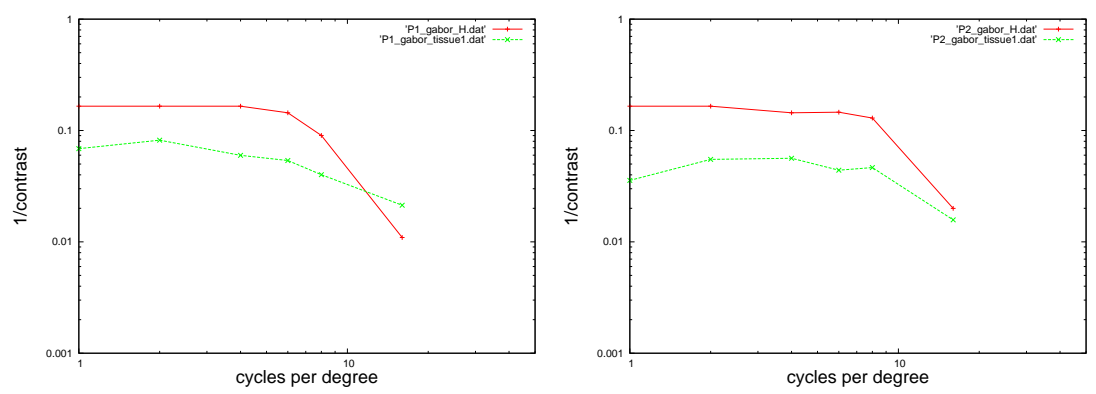

Fig. 3. Relation of the CSF for homogeneous background to CSF for tissue background; left: results of observer P1; right: results of observer P2
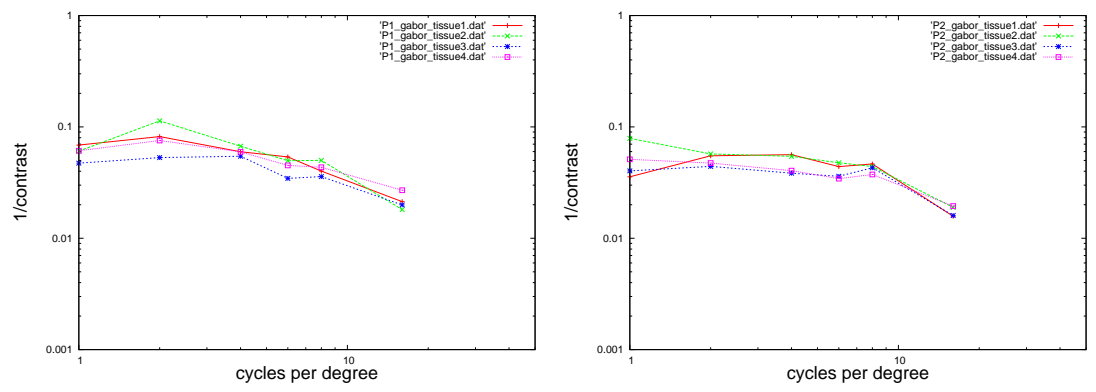

Fig. 4. CSF for 4 different mammographic images (cp. Fig. 2); left: results of observer $\mathrm{P} 1$; right: results of observer $\mathrm{P} 2$
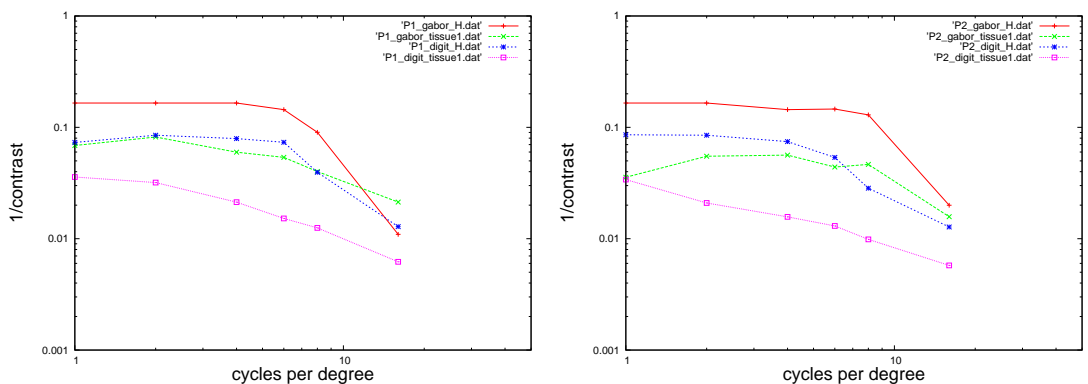

Fig. 5. Relation between CSF for digits to CSF for Gabor pattern on homogeneous and tissue background; left: results of observer P1; right: results of observer P2 
The run of the CSF curve is similar for mammograms with different tissue characteristics (Fig. 4). An analysis of the image parts with a quadrature filter showed a similar frequency distribution. It is striking that the CSF is slightly higher on radiolucent tissue, which appears more homogeneous than the other tested tissues (bright curve in Fig. 4). The results of the eight observers confirm the second hypothesis, and let assume that an analysis of the CSF for a tissue background is slightly depending on the selected mammographic image.

As expected, the CSF is higher for the discrimination task than for the identification task (Fig. 5). For the identification of the digits higher cognitive processes are required. This is evident for both a homogeneous background as well as for a tissue background.

Studying the CSF in a mammographic context opens new possibilities for a psychophysical analysis of the contrast sensitivity depending on individual preferences, ambient conditions in the reading room, visualization methods and the design of application, e.g. with regard to luminance distributions in the field of view. This may support the diagnostic certainty in reporting breast cancer.

\section{References}

1. Barten PGJ. Contrast Sensitivity of the Human Eye and Its Effects on Image Quality. SPIE Press Monograph Vol. PM72; 1999.

2. Fletcher-Heath L, Metter RV. Quantifying the performance of human and software CDMAM phantom image observers for the qualification of digital mammography systems. Proc SPIE. 2005; p. 486-98.

3. Burgess AE, Jacobson FL, Judy PF. Mass discrimination in mammography: Experiments using hybrid images. Acad Radiol. 2003;10:1247-56.

4. Kaernbach C. Adaptive threshold estimation with unforced-choice tasks. Percept Psychophys. 2001;63(8):1377-88. 